\title{
Scanning Convergent Beam Electron Diffraction (CBED), the Essential Questions of Why, What and How?
}

\author{
Jian-Min Zuo and Yu-Tsun Shao
}

Department of Materials Science and Engineering and Fredrick Seitz Materials Research Laboratory, University of Illinois at Urbana-Champaign, Urbana, Illinois, USA

CBED is recorded using a focused electron probe at the specimen [1]. The probe convergence angle in CBED is several times larger than what is used in nanobeam diffraction (NBD), but it is still significantly smaller than the convergence angle used in an aberration corrected STEM. The relatively large convergence angle used for CBED gives rise to transmitted and diffracted disks (see Fig. 1 for an example); the size of the disk determines the range of excitation errors $\left(S_{\mathrm{g}}\right)$ for each reflection. The intensity versus $S_{\mathrm{g}}$, or rocking curve information, recorded in CBED is used for crystal symmetry determination and quantitative structure factor measurement. CBED is also used to record high order Laue zone (HOLZ) lines, which provide accurate measurement of crystal lattice parameters or electron acceleration voltage.

Scanning CBED (SCBED) is an extension of CBED. Using the deflection coils, scanning CBED patterns can be recorded from an area of the sample for every probe positions, to provide spatially resolved structural information. This can be done either in a TEM or STEM. Diffraction patterns are recorded using a 2D digital detector, for example, a CCD camera. Compared to CBED, which records one diffraction pattern over one probe position, SCBED collects the full 4-D data, in the form of two spatial coordinates, the $(x, y)$ in the real space and the $\left(k_{x}, k_{y}\right)$ in the reciprocal space. SCBED differs from scanning electron nanodiffraction (SEND) by the beam convergence angle (which is larger for SCBED).

Why SCBED? SCBED works like STEM, but by recording the whole diffraction pattern, SCBED can be analyzed to extract quantitative structure information, which is simply not possible with BF-STEM, ADFSTEM or differential phase contrast (DFC) STEM. One example is mapping of crystal symmetry in ferroelectric crystals. Figure 1 shows an application of the symmetry mapping in $(1-\mathrm{x}) \mathrm{Pb}\left(\mathrm{Zn}_{1 / 3} \mathrm{Nb}_{2 / 3}\right) \mathrm{O}_{3}-$ $\mathrm{xPbTiO}_{3}(\mathrm{x}=0.08)(\mathrm{PZN}-\mathrm{PT})$ [2]. The change in CBED pattern symmetry was quantified using the normalized cross-correlation $(\gamma)$ value of a pair of diffraction discs related by mirror symmetry. The highest $\gamma$ values are detected along two different directions (A and B) as shown in Figure 1(b) and (d). The $\mathrm{A}$ and $\mathrm{B}$ directions are rotated by $45^{\circ}$ along the $[100]_{C}$ zone axis. The corresponding simulated patterns identifies monoclinic $P m$ zone axis $[100]_{P m}$ and $[010]_{P m}$, as the crystal orientation.

What information does SCBED provide? Structural information obtainable from SCBED includes: 1) The disc position can be used to measure the d-spacing of individual reflections; 2) Pattern symmetry and the dynamic extinction can be used to map the crystal symmetry or the lack of symmetry; 3 ) The change in diffraction intensity can be used to identify structural defects; 4) Electron diffraction intensity can be used to determine the atomic positions; 5) accurate determination of lattice parameters and strain (small strain) using HOLZ lines; and 6) accurate structure factor measurement from diffraction intensities can be used to determine the atomic thermal vibrations (the Debye-Waller factors), and crystal potential or charge density.

How to do SCBED? SCBED can be carried out by first selecting an area of interest, dividing this area into a number of pixels, placing the electron probe at each of these pixels and recording the diffraction patterns 
at each pixel using an electron camera. Data acquisition is automated using either a dedicated hardware to synchronize the scan and diffraction pattern recording or by using computer control of the TEM and the electron camera. An implementation of SCBED using the second approach is reported by Kim et al. [3]. This technique does not require additional hardware other than the computer and the electron detector that are already installed on the TEM. The main drawback is that the speed of acquisition is limited by the camera readout speed or the speed of beam deflection inside the TEM, whichever is slower. Removal of the inelastic background is critical for quantitative analysis of SCBED, which can be done by using an electron energy-filter [1]. The filtering also helps the analysis of thick crystals by removing the inelastic background. Quantitative intensity analysis further requires the correction of diffraction pattern distortion, detector point spread function and care of sample preparation [4-6].

This talk will review what we have learnt before about quantitative CBED and applications of SCBED. Emphasis will be on strategies to optimize the acquisition and analysis of SCBED. New algorithms deal with the large dataset of SCBED will also be discussed [7].

References:

[1] Zuo, J.M. and Spence, J.C.H., "Advanced Transmission Electron Microscopy". 2017: Springer.

[2] Y. T. Shao and J. M. Zuo, Physical Review Letters 118(15) (2017), p. 157601.

[3] Kim, K.H. et al, Micron 71 (2015), p. 39-45.

[4] J. M. Zuo, Reports on Progress in Physics 67(11) (2004), p. 2053.

[5] K. Tsuda and M. Tanaka, Acta Crystallographica Section A 55 (1999), p. 939.

[6] Ding Peng and Philip N. H. Nakashima, Journal of Applied Crystallography 50(2) (2017), p. 602.

[7] Supported by U.S. DoE, Office of Basic Energy Sciences under contract DEFG02-01ER45923.
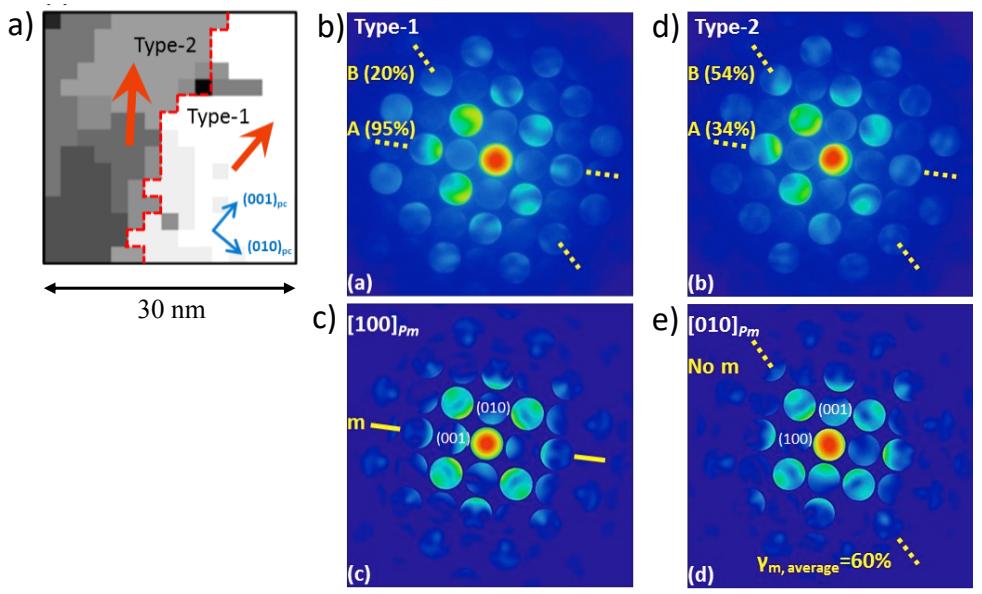

Figure 1. Determination of nanodomains in $(1-\mathrm{x}) \mathrm{Pb}\left(\mathrm{Zn}_{1 / 3} \mathrm{Nb}_{2 / 3}\right) \mathrm{O}_{3}-\mathrm{xPbTiO}_{3}(\mathrm{x}=0.08)$ using SCBED. (a) the mirror symmetry as measured by $\gamma$ varies across two types of domains. The red dashed line indicates the domain boundary. The orange arrows indicate the projected polarization directions for each type of domain. The polarization directions were determined with help of simulations. Figures in b) to d) show experimental and simulated CBED patterns. The mirror plane in the (b) type-1 and (d) type-2 domains is rotated by $45^{\circ}$. Figs. (c) \& (e) show simulated patterns using the Bloch wave method assuming Pm phase symmetry. 